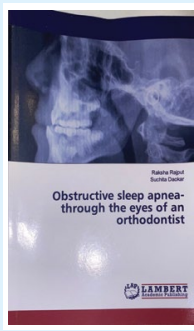

\title{
OBSTRUCTIVE SLEEP APNEA - THROUGH THE EYES OF AN ORTHODONTIST
}

Raksha Rajput and Suchita Daokar; 2019; LAP LAMBERT Academic Publishing; $€ 39.90$; pp. 76:

ISBN: 978-613-9-45259-0

Obstructive sleep apnoea (OSA) is defined as 'recurrent upper airway obstruction during sleep', which can have a detrimental impact on the health and wellbeing of those affected by it. As it is often underdiagnosed, and dentists may be the first to recognise signs and symptoms, this book is a welcomed resource to revise the theory and current approaches to manage this disorder whilst also considering the role of the orthodontist in its treatment.

Obstructive sleep apnea - through the eyes of an orthodontist provides a concise, simplified guide which discusses the definition, aetiology, investigations and management options for this condition. It also covers how orthodontic treatment can help manage OSA in a non-invasive way and the potential complications that can arise if it is untreated. This publication is aimed at orthodontists and dental professionals with an orthodontic background, who have a role in treating OSA.

Despite the short length of this text, the relevant aspects are covered in adequate detail to engage readers and earn its place on the bookshelf of practitioners with a particular interest in OSA. It is easy to read, comprised of 11 chapters which are categorised further with sub-headings. The initial chapters cover the theory of the condition, whilst the latter parts delve into the diagnosis using cephalometrics and management, covering both non-surgical medical approaches and non-medical options.

The two main orthodontic approaches to manage this condition are discussed in more detail: tongue-raising appliances and mandibular advancement devices. Whilst the authors utilise clinical photographs to show how these devices work, the images are black and white and somewhat pixelated making interpretation difficult. Understanding the mechanics of how these appliances work can be challenging with two-dimensional images. Perhaps future editions could incorporate either coloured diagrams or links to interactive animations which would better accompany clinical descriptions within the text.

Overall, this book is a comprehensive resource for dentists and orthodontists with a particular interest in this disorder in providing a brief overview of the diagnosis and management options for this condition. As it is only 66 pages in length, it covers the key points succinctly and this could facilitate its use as a quick and effective reference guide in clinical settings. However, for practitioners and orthodontists who are experienced in managing OSA and perhaps looking for an advanced text, this book may lack detail and depth and could be perceived as an over-simplistic explanation of a complex condition.

Emma Carr 